\title{
Mulberry EIL3 confers salt and drought tolerances and modulates ethylene biosynthetic gene expression
}

\author{
Changying Liu ${ }^{1}$, Jun Li ${ }^{2}$ ，Panpan Zhu ${ }^{1,3}$ ， Jian Yu ${ }^{1}$ ， Jiamin Hou ${ }^{1}$ ，Chuanhong Wang ${ }^{4}$ ， Dingpei Long ${ }^{1}$, \\ Maode Yu ${ }^{1}$, Aichun Zhao ${ }^{\text {Corresp. } 1}$ \\ ${ }^{1}$ State Key Laboratory of Silkworm Genome Biology, Key Laboratory of Sericultural Biology and Genetic Breeding, Chongqing, China \\ 2 Guiyang University of Chinese Medicine, Guiyang, China \\ 3 Bioengineering College of Chongqing University, Chongqing, China \\ 4 The National Key Engineering Lab of Crop Stress Resistance Breeding, School of Life Sciences, Anhui Agricultural University, Hefei, China \\ Corresponding Author: Aichun Zhao \\ Email address: zhaoaichun@hotmail.com
}

Ethylene regulates plant abiotic stress responses and tolerances, and ethyleneinsensitive3 (EIN3)/EIN3-like (EIL) proteins are the key components of ethylene signal transduction. Although the functions of EIN3/EIL proteins in response to abiotic stresses have been investigated in model plants, little is known in non-model plants, including mulberry (Morus L.), which is an economically important perennial woody plant. We functionally characterized a gene encoding an EIN3-like protein from mulberry, designated as MnEIL3. A quantitative real-time PCR analysis demonstrated that the expression of MnEIL3 could be induced in roots and shoot by salt and drought stresses. Arabidopsis overexpressing MnEIL3 exhibited an enhanced tolerance to salt and drought stresses. MnEIL3 overexpression in Arabidopsis significantly upregulated the transcript abundances of ethylene biosynthetic genes. Furthermore, MnEIL3 enhanced the activities of the MnACO1 and MnACS1 promoters, which respond to salt and drought stresses. Thus, MnEIL3 may play important roles in tolerance to abiotic stresses and the expression of ethylene biosynthetic genes. 


\section{Mulberry EIL3 confers salt and drought tolerances and}

\section{2 modulates ethylene biosynthetic gene expression}

3 Changying $\mathrm{Liu}^{1}$, Jun $\mathrm{Li}^{2}$, Panpan $\mathrm{Zhu}^{1,3}$, Jian $\mathrm{Yu}^{1}$, Jiamin $\mathrm{Hou}^{1}$, Chuanhong Wang ${ }^{4}$, Dingpei

4 Long $^{1}$, Maode $\mathrm{Yu}^{1}$ and Aichun $\mathrm{Zhao}^{1}$

51 State Key Laboratory of Silkworm Genome Biology, Key Laboratory of Sericultural Biology

6 and Genetic Breeding, Southwest University, Chongqing 400716, P.R. China.

72 Guiyang University of Chinese Medicine, Guiyang 550025, P. R. China.

83 Bioengineering College of Chongqing University, Chongqing 400044, P. R. China.

94 The National Key Engineering Lab of Crop Stress Resistance Breeding, School of Life

10 Sciences, Anhui Agricultural University, Hefei 230036, P. R. China.

11 Corresponding author

12 Aichun Zhao, zhaoaichun@hotmail.com or zhaoaichun@swu.edu.cn 
14 ABSTRACT

Ethylene regulates plant abiotic stress responses and tolerances, and ethylene-insensitive3 (EIN3)/EIN3-like (EIL) proteins are the key components of ethylene signal transduction. Although the functions of EIN3/EIL proteins in response to abiotic stresses have been investigated in model plants, little is known in non-model plants, including mulberry (Morus L.), which is an economically important perennial woody plant. We functionally characterized a gene encoding an EIN3-like protein from mulberry, designated as MnEIL3. A quantitative real-time PCR analysis demonstrated that the expression of MnEIL3 could be induced in roots and shoot by salt and drought stresses. Arabidopsis overexpressing MnEIL3 exhibited an enhanced tolerance to salt and drought stresses. MnEIL3 overexpression in Arabidopsis significantly upregulated the transcript abundances of ethylene biosynthetic genes. Furthermore, MnEIL3 enhanced the activities of the MnACOI and MnACS1 promoters, which respond to salt and drought stresses. Thus, MnEIL3 may play important roles in tolerance to abiotic stresses and the expression of ethylene biosynthetic genes.

Key words: Mulberry, MnEIL3, Arabidopsis, Abiotic stresses, Salt stress, Drought.

\section{INTRODUCTION}

Ethylene is gaseous hormone that regulates many physiological processes, including seed germination, seedling growth, leaf expansion, flower opening, senescence, and fruit development. Ethylene is synthesized from methionine by a catalysis mediated by S-adenosyl-L-methionine synthetase, 1-aminocyclopropane-1-carboxylic acid synthase (ACS), and 1-aminocyclopropane1-carboxylic acid oxidase (ACO) (Kende, 1993; Yang \& Hoffman, 1984). ACS and ACO are the rate-limiting enzymes that regulate ethylene biosynthesis, and several regulators influence ethylene production by changing the activities and expression levels of $A C S$ and $A C O$.

The ethylene perception and signal transduction pathway has been well studied in model plants, like Arabidopsis thaliana, tomato (Solanum lycopersicum), and tobacco (Nicotiana tabacum). In the presence of ethylene, the endoplasmic reticulum-localized receptors (ethylene 
40

41

42

receptors, ETRs) perceive ethylene, resulting in the inactivation of constitutive triple response 1, which is the negative regulatory factor of ethylene responses (Clark et al., 1998; Hua et al., 1998; Rodríguez et al., 1999). Then, ethylene insensitive 2 (EIN2), an essential positive regulator of ethylene signaling, is dephosphorylated, and its carboxyl terminus is cleaved and enters into the nucleus, where it binds to ethylene insensitive 3/EIN3-like proteins (EIN3/EILs) (Chao et al., 1997; Qiao et al., 2012). Eventually, the activated EIN3/EILs regulate the transcription of ethylene-responsive factors (ERFs) and other downstream genes (Alonso \& Stepanova, 2004; Wang et al., 2002).

EIN3/EILs are the key elements that initiate the ethylene-mediated downstream transcriptional cascade (An et al., 2018). The mutation of EIN3/EILs genes, AtEIN3 (AT3G20770) and AtEIL1 (AT2G27050), result in ethylene-insensitive performance, and plants overexpressing AtEIN3 and AtEIL1 show enhanced ethylene production and triple responses in Arabidopsis. The ein3-1 eill-1 double mutant completely abolishes the ethylene response in etiolated Arabidopsis seedlings (Chao et al., 1997; Alonso et al., 2003). The stabilities of EIN3/EILs are regulated by EIN3-binding F-box proteins (EBF1 and EBF2) in the EBF1- and EBF2-mediated ubiquitin-proteasome degradation pathway, and mutations of $E B F 1$ and $E B F 2$ accumulate EIN3/EIL proteins and display constitutive ethylene responses (Potuschak et al., 2003). Ethylene quickly stabilizes EIN3/EIL1 by promoting EBF1 and EBF2 proteasomal degradation, which contributes to the ethylene responses (An et al., 2010). In addition, the MKK9-MPK3/MPK6 cascades promote EIN3-mediated transcription in ethylene signaling by regulating the phosphorylation and protein stability of EIN3 (Yoo et al., 2008).

The EIN3/EILs family are plant-specific transcription factors (TFs) and bind to primary ethylene response elements (PEREs) and EIL conserved binding sequences (ECBSs) in the promoters of downstream genes involved in the response to ethylene (Yin et al., 2010). Thus, EIN3/EILs regulate many physiological processes, including apical hook formation, hormone responses, fruit development, abiotic stress responses, seedling photomorphogenesis, and light perception, by activating the expression of a wide range of downstream genes (An et al., 2012; 
67

68

He et al., 2011; Zhu et al., 2011; Shan et al., 2012; Peng et al., 2014; Shi et al., 2012; Shi et al., 2018). Recently, studies have focused on the functions of EIN3/EILs in abiotic stress tolerances. Peng et al. (2014) demonstrated that EIN3/EIL1 are essential for the enhanced ethylene-induced salt tolerance in Arabidopsis, and salt stress stabilizes EIN3/EIL1 proteins by promoting EBF1/EBF2 proteasomal degradation in an EIN2 independent manner. In addition, a large number of EIN3/EIL1-regulated genes that participate in salt stress responses have been identified using whole-genome transcriptome analyses, including many genes encoding reactive oxygen species scavengers. An AP2 domain-containing gene, ESE1, is an ethylene-modulated gene downstream of EIN3/EIL1 in the salt response (Zhang et al., 2011). Mutations of EIN3 increase the sensitivity in response to water stress stimulated by polyethylene glycol (PEG) 6000 (Cui et al., 2015). Genetic and biochemical analyses revealed that EIN3 proteins act as negative factors against freezing stress by repressing the expression of C-repeat binding factors and typeA Arabidopsis response regulator (ARR) 5, ARR7, and ARR15 (Shi et al., 2012). The functions of the EIN3/EIL1 proteins in response to heavy metal stresses have also been studied. Kong et al (2018) found that cadmium (Cd) inhibits EIN3 protein degradation in Arabidopsis, and the ein31 eill-1 double mutant plants display an increased tolerance to $\mathrm{Cd}$. EIN3 enhances root growth inhibition under $\mathrm{Cd}$ stress by regulating the expression of the xyloglucan endotransglucosylase/hydrolase 33 and response to low sulfur 1 genes, which are involved in cell wall modification and sulfur metabolic processes, respectively (Kong et al., 2018).

Mulberry (Morus L.) is an economically important perennial woody plant belonging to Moraceae of Rosales, which have multiple uses in silkworm rearing, ecology, pharmaceuticals, and traditional Chinese medicines (He et al., 2013). Mulberry adapts well to drought, salinity, water logging, and other abiotic stress conditions, but little is known regarding the molecular mechanisms of the tolerance. In our previous studies, the elements involved in mulberry ethylene biosynthesis and signal transduction were identified and its functions in fruit development were clarified (Liu et al., 2014; Liu et al., 2015). However, the functions of mulberry ethylene biosynthesis and signal pathway related genes in other aspects of the lifecycle remain unclear, 
94

95

96

especially in abiotic stress responses and tolerances. In this study, we investigated the physiological functions of a mulberry gene encoding EIN3-like proteins, MnEIL3, in salt and drought tolerances by analyzing its expression patterns and its heterologous overexpression in Arabidopsis. MnEIL3's expression was significantly upregulated by salt and drought stresses, and its overexpression in Arabidopsis led to enhanced salt and drought stress tolerances and the upregulated expression of ethylene biosynthetic genes. Furthermore, MnEIL3 significantly enhanced the activities of $M n A C O 1$ and MnACS1 promoters. Thus, a working model for MnEIL3 in plant tolerance to abiotic stresses was suggested.

\section{MATERIALS AND METHODS}

\section{Plant materials and growth conditions}

A. thaliana ecotype Columbia-0 and the ein3-1 eill-1 mutant were used as plant materials and were grown at $24^{\circ} \mathrm{C} / 22^{\circ} \mathrm{C}$ under a 16 -h light/8-h dark photoperiod.

A mulberry (M. notabilis Schneid) tree, which was used for genome sequencing, is an isolated wild mulberry species with a chromosome number of 14 . The seedlings of M. notabilis were used in this study and grown in a PQX-type plant incubator with artificial intelligence capability (Ningbo Southeast Instrument Corporation, China) under a 16-h light/ 8-h dark photoperiod at $26^{\circ} \mathrm{C} / 22^{\circ} \mathrm{C}$ (day/night). For stress treatments, the one-month-old seedlings were subjected to salt $[0.6 \%(\mathrm{~m} / \mathrm{v}) \mathrm{NaCl}]$ and drought $[20 \%(\mathrm{~m} / \mathrm{v})$ PEG6000]. The roots and shoot of the treated seedlings were sampled at $0,1,3,6,12$, and $24 \mathrm{~h}$ post-treatment. The 14-d-old seedlings were treated independently with $200 \mathrm{mM} \mathrm{NaCl}$ and $200 \mathrm{mM}$ mannitol, and the treated seedlings were sampled at $0,1,3,6$, and $12 \mathrm{~h}$ post-treatment. The harvested materials were frozen immediately in liquid nitrogen for total RNA extraction.

\section{RNA extraction and quantitative real-time PCR (qRT-PCR)}

Total RNA extraction, first-strand of cDNA synthesis, and qRT-PCR analysis were performed as described in our previous study (Wei et al., 2014). The ACTIN3 and $\beta$-actin2 genes were used as internal controls for mulberry and Arabidopsis, respectively, and the relative expression was 
120

121

122

123

124

125

126

127

128

129

130

131

132

133

134

135

136

137

138

139

140

141

142

143

144

145

defined as $2^{-[\mathrm{Ct}(\text { target gene) } \mathrm{Ct}(\mathrm{control} \text { gene) })}$. All qRT-PCRs were performed with three independent biological replicates. The primers used are specified in Table S2.

\section{Plasmid construction}

The full-length coding sequence of MnEIL1 (GenBank accession number: XM_010107825) and MnEIL3 (XM_010093690) were cloned into the NcoI and BglII restriction sites of the pCAMBIA1302 expression vector under the control of the CaMV35S promoter, and MnEIL2 (XM_010107826) was cloned into the BgIII and SpeI restriction sites of the pCAMBIA1302 expression vector. Finally, the CaMV35S::MnEIL1, CaMV35S::MnEIL2, and CaMV35S::MnEIL3 recombinant plasmids were generated.

The $5^{\prime}$ upstream regions of the MnACS1 and MnACS3 genes were independently inserted into the EcoRI and NcoI restriction sites of the pCAMBIA1301 expression vector, producing the MnACS1pro::GUS and MnACS3pro::GUS recombinant plasmids, respectively. The primers used are specified in Table S2.

\section{Plant transformation}

The recombinant plant expression vectors were transformed into Agrobacterium tumefaciens strain GV3101. MnEIL3, MnACS1pro::GUS and MnACS3pro::GUS were eventually independently transformed into A. thaliana (Columbia-0) using the floral dip method (Clough \& Bent, 1998). The transgenic Arabidopsis lines were evaluated by GUS staining, genomic PCR, inverse PCR, and qRT-PCR analyses. The homozygous lines of the T3 generation were used for further research.

\section{Stress treatments of transgenic Arabidopsis}

Wild type, ein3-1 eill-1, and MnEIL3 transgenic seeds were germinated on 1/2 Murashige and Skoog (MS) agar medium. The 7-d-old seedlings were transferred into pots containing the soil supplemented with normal nutrients were grown at $24^{\circ} \mathrm{C} / 22^{\circ} \mathrm{C}$ under a 16 -h light $/ 8$-h dark photoperiod. The 21- and 14-d-old seedlings were treated with salt $[1.2 \%(\mathrm{~m} / \mathrm{v}) \mathrm{NaCl}]$ and drought (watering treatments withheld), respectively, and the proline, hydrogen peroxide $\left(\mathrm{H}_{2} \mathrm{O}_{2}\right)$, 
146

147

148

149

150

151

152

153

154

155

156

157

158

159

160

161

162

163

164

165

166

167

168

169

170

171

and malondialdehyde (MDA) contents, were measured using their respective test kits (Jiancheng Bioengineering Institute, Nanjing, China) according to the manufacturer's instructions. Each treatment was replicated three times.

The seeds of the transgenic plants that contained MnACS1pro::GUS and MnACS3pro::GUS were germinated on 1/2 MS agar medium. The 10-d-old seedlings were exposed to salt (200 mM $\mathrm{NaCl})$ and drought (200 mM mannitol) treatments. The samples were subjected to GUS staining after $0,1,3,6$, and $12 \mathrm{~h}$ of exposure.

\section{Transient expression assays and GUS activity detection}

CaMV35S::MnEIL1, CaMV35S::MnEIL2, and CaMV35S::MnEIL3 recombinant plasmids were used as effector plasmids, and MnACO1pro::GUS, MnACO2pro::GUS, MnACS1pro::GUS, and MnACS3pro::GUS recombinant plasmids were used as reporter plasmids. Of these vectors, the MnACO1pro::GUS and MnACO2pro::GUS recombinant plasmids have been reported in a previous study (Yu et al., 2017). The reporter and effector plasmids were transformed into $A$. tumefaciens strain GV3101. The bacteria were mixed and co-injected into the strawberry fruit as described in a previous study (Spolaore et al., 2001). The injected tissues were sampled and used for the GUS staining analysis. Meanwhile, the GUS activity of the injected tissues were detected by 4-nitrophenyl- $\beta$-D-glucopyranoside methods (Jefferson, 1987).

\section{Statistical analyses}

The statistical analyses methods were as described in a previous study (Liu et al., 2017a). All data were conducted using SPSS statistical software 17.0 (SPSS Inc., Chicago, IL, USA) and Excel 2013 (Microsoft, Redmond, CA, USA). The results are presented as mean values \pm SEs. The significant differences between samples were analyzed using a one-way ANOVA in SPSS Statistics 17.0. The analyses of significant differences $(P<0.05)$ were measured by Student's $t$ test analysis.

\section{RESULTS}

Expression patterns of $M n E I L$ genes under salt and drought stresses 
172 The expression levels of MnEIL genes under $\mathrm{NaCl}$ and PEG treatments were assessed by qRT173 PCR. Under salt stress, the transcript abundance of MnEIL1 was significantly upregulated and 174 downregulated in roots and shoots, respectively, $3 \mathrm{~h}$ after the $\mathrm{NaCl}$ treatment, but the expression 175 levels after $24 \mathrm{~h}$ were not different than those at $0 \mathrm{~h}$ (Figs. 1A and 1B). The expression of

MnEIL2 in roots was significantly upregulated after $1 \mathrm{~h}$ of $\mathrm{NaCl}$ treatment. MnEIL2's expression in shoots was downregulated at 1, 6, and $12 \mathrm{~h}$, but its expression was upregulated at $24 \mathrm{~h}$ after the $\mathrm{NaCl}$ treatment (Figs. 1A and 1B). The transcript abundances of MnEIL3 in roots and shoot were significantly upregulated $3 \mathrm{~h}$ after the $\mathrm{NaCl}$ treatment, but the expression of MnEIL3 in shoots after $24 \mathrm{~h}$ showed no difference with that at $0 \mathrm{~h}$ (Figs. 1A and 1B). Under drought stress conditions, MnEIL1's expression levels in roots and shoot were downregulated by the $\mathrm{NaCl}$ treatment, although MnEIL1's expression in roots was upregulated at $3 \mathrm{~h}$ (Figs. 1C and 1D). The transcript abundance of MnEIL2 was significantly upregulated and downregulated after $3 \mathrm{~h}$ of $\mathrm{NaCl}$ treatment in roots and shoot, respectively (Figs. 1C and 1D). The expression of MnEIL3 in roots was significantly upregulated after a PEG treatment, although its expression showed no response to PEG at $12 \mathrm{~h}$. MnEIL3's expression in shoots was significantly upregulated $6 \mathrm{~h}$ after the PEG treatment and exhibited a strong expression peak at $24 \mathrm{~h}$ (Figs. 1C and 1D).

\section{The overexpression of MnEIL3 in Arabidopsis enhances salt and drought tolerances}

The MnEIL3 gene was selected for further investigation base on its responses to salt and drought stresses. The full-length sequence of the MnEIL3 gene was inserted into the pCAMBIA1302 vector under the control of the CaMV35S promoter and transformed into wild type Arabidopsis plants. Transgenic lines were obtained using hygromycin resistance and confirmed by genomic PCR and qRT-PCR analyses (Figs. S1A and S1B). In addition, the insertion site of the transgene construct was determined using inverse PCR. The CaMV35S::MnEIL3 recombinant plasmid was inserted into chromosome 2 of the Arabidopsis genome (Fig. S1C).

To evaluate the tolerance of MnEIL3-overexpressing (MnEIL3ox) plants against salt stress, the 21-d-old wild type, MnEIL3ox, and ein3-1 eil1-1 Arabidopsis seedlings were treated with 1.2\% $(\mathrm{m} / \mathrm{v}) \mathrm{NaCl}$. After treatment, MnEIL3ox plants showed relatively greater growth rates than wild 
199

200

201

202

203

204

205

206

207

208

209

210

211

212

213

214

215

216

217

218

219

220

221

222

223

224

225

type and ein3-1 eil1-1 Arabidopsis. Additionally, the ein3-1 eill-1 seedlings showed a decreased salt tolerance compared with wild type plants (Figs. 2A and 2B). To characterize the performance of MnEIL3ox plants under drought stress, the 14-d-old seedlings of wild type, MnEIL3ox, and ein3-1 eil1-1 Arabidopsis plants were treated with drought stress. The growth of wild type, MnEIL3ox, and ein3-1 eill-1 plants showed no difference under drought stress conditions. However, MnEIL3ox showed a greater capability to survive than wild type and ein31 eil1-1 plants when the treated plants were re-watered (Figs. 2A and 2B).

To understand the mechanism behind the enhanced sensitivity to drought and salt stresses caused by MnEIL3's overexpression, the accumulated levels of $\mathrm{H}_{2} \mathrm{O}_{2}, \mathrm{MDA}$, and proline were analyzed. The MDA and $\mathrm{H}_{2} \mathrm{O}_{2}$ contents in MnEIL3ox and ein3-1 eil1-1 plants were lower and higher than in wild type Arabidopsis, respectively (Figs. 2C and 2D). The proline contents in MnEIL3ox and ein3-1 eill-1 plants were higher and lower than in wild type Arabidopsis, respectively (Figs. 2C and 2D). Thus, MnEIL3 may negatively regulate drought and salt stress tolerances.

\section{The enhanced expressions of $A C S$ and $A C O$ genes in MnEIL3ox plants}

In this study, the expression levels of ACS- and ACO-encoding genes were detected in MnEIL3ox plants. The transcript abundances of AtACS4/6/8/10/12 genes in MnEIL3ox plants were higher than in wild type Arabidopsis, while the expression levels of AtACS1/7 genes showed were lower in MnEIL3ox plants. Moreover, there was no difference in the expression of AtACS3 between MnEIL3ox and wild type plants (Fig. 3). All of the detected AtACO genes, AtACO1/2/4, showed higher expression levels in MnEIL3ox plants than in wild type Arabidopsis (Fig. 3).

Mulberry EIL proteins modulate $M n A C O 1, M n A C O 2, M n A C S 1$, and $M n A C S 3$ promoter activities

To explore the correlations between the expression of MnEIL3 and ethylene biosynthetic genes, $M n A C O 1, M n A C O 2, M n A C S 1$, and $M n A C S 3$ were selected for promoter isolation. All these genes have been determined as the key genes involved in ethylene biosynthesis in mulberry (Liu 
226

227

228

229

230

231

232

233

234

235

236

237

238

239

240

241

242

243

244

245

246

247

248

249

250

251

252

et al., 2014; Liu et al., 2015). The gene' promoters were downloaded from the Morus genome database (http://morus.swu.edu.cn/morusdb/) and isolated from mulberry (M. notabilis). By searching for cis-acting regulatory elements, PERE- and ECBS-binding sites were identified in the promoters of MnACO1, MnACO2, MnACS1, and MnACS3 (Table S1). In vivo interactions between MnEIL3 and these promoters were estimated by transient analyses in strawberry fruit. MnEIL3 significantly enhanced the activities of MnACO1 and MnACS1 promoters, while no significant effects on the activities of MnACO2 and MnACS3 promoters were found (Fig. 4). Thus, EIL proteins may act as the transcriptional activators of ethylene biosynthetic genes. We also detected correlations between the other two MnEIL genes, MnEIL1/2, and ethylene biosynthetic genes. Thus, MnEIL1 acted as the activator of MnACO2 and MnACS3 promoters, while MnEIL2 regulated the activities of the MnACO1 and MnACS3 promoters (Fig. 4).

The activities of $M n A C S 1$ and $M n A C S 3$ promoters were regulated by salt and drought stresses

To examine the responsiveness of the MnACS1 and MnACS3 genes under salt and drought stresses, transgenic Arabidopsis were generated by introducing MnACS1pro::GUS- and MnACS3pro::GUS-fused genes, and then the 10-d-old seedlings were exposed to stresses (Fig. 5A). The MnACS1 promoter in leaves responded to $\mathrm{NaCl}$ and mannitol treatments (Fig. 5A). The GUS reporter in the MnACS3pro::GUS transgenic Arabidopsis was mainly expressed in roots, while little GUS accumulation levels in stems and leaves were detected. Under salt stress, the GUS accumulation levels in the stems of MnACS3pro::GUS transgenic Arabidopsis were enhanced after $6 \mathrm{~h}$ of $\mathrm{NaCl}$ treatment, while the GUS levels in stems and leaves were enhanced after $3 \mathrm{~h}$ of mannitol treatment (Fig. 5A).

\section{The expression levels of $M n A C S 1$ and $M n A C S 3$ are regulated by salt and drought stresses}

The 14-d-old seedlings were treated with $\mathrm{NaCl}$ and mannitol, and then used to detect the expression levels of $M n A C S 1$ and $M n A C S 3$. MnACS1's expression level was significantly upregulated after $1 \mathrm{~h}$ of $\mathrm{NaCl}$ treatment, and its expression was transiently upregulated after $1 \mathrm{~h}$ of mannitol treatment and then was downregulated (Figs. 5B and 5C). The transcript abundance 
253

254

255

256

257

258

259

260

261

262

263

264

265

266

267

268

269

270

271

272

273

274

275

276

277

278

279

of $M n A C S 3$ was significantly upregulated after $3 \mathrm{~h}$ of $\mathrm{NaCl}$ treatment, and its expression was upregulated after $3 \mathrm{~h}$ of mannitol treatment, but it showed no response at any other time point (Figs. 5D and 5E).

\section{DISCUSSION}

EIN3/EILs proteins are positive factors in ethylene signal transduction. In model plants, EIN3/EILs are involved in many aspects of the life cycle, including seed germination, soil emergence, seedling development, leaf senescence, pigments biosynthesis, light perception, and abiotic stress responses (Kim et al., 2017; Zhong et al., 2014; An et al., 2018; Yu et al., 2013; Yu et al., 2016). Additionally, the regulatory functions of EIN3/EILs in response to abiotic stresses have also attracted considerable attention (Zhang et al., 2011; Cui et al., 2015; Shi et al., 2012; Kong et al., 2018; Peng et al., 2014). However, there are no reports on the functions of EIN3/EILs in the abiotic stress tolerance of woody plants, including mulberry. In the present study, the expression levels of mulberry MnEIL genes under salt and drought stresses were revealed, and they showed different patterns. Among these genes, the expression of MnEIL3 was significantly upregulated by salt and drought stresses in roots and shoots (Fig. 1), which is similar to the expression patterns of Arabidopsis AtEIN3 and AtEIL1 genes (Fig. S2). The fulllength coding sequence of MnEIL3 was transformed into Arabidopsis for stress tolerance analysis. The expression levels of MnEIL3 were significantly upregulated in roots and shoots by $\mathrm{NaCl}$ treatments (Fig. 1), and the overexpression of this gene in Arabidopsis enhanced salt stress tolerance (Fig. 2). MnEIL3's overexpression decreased the MDA and $\mathrm{H}_{2} \mathrm{O}_{2}$ contents and enhanced the proline content under salt stress. Thus, MnEIL3 positively regulated plant salt tolerance, which was similar to the results described by Peng et al (2014). In this study, ein3-1 eill-1 plants showed decreased tolerances to drought stress compare with wild type plants (Fig. 2), and mutation of EIN3/EILs decreased the MDA and $\mathrm{H}_{2} \mathrm{O}_{2}$ contents and enhanced the proline content under drought stress. This was similar to the results of a previous study which reported that the ein3-1 mutant exhibited a decreased tolerance to drought stress stimulated by PEG6000 (Cui et al., 2015). In addition, MnEIL3ox plants showed a greater ability to survive drought 
280

281

282

283

284

285

286

287

288

289

290

291

292

293

294

295

296

297

298

299

300

301

302

303

304

305

306

stress than wild type and ein3-1 eill-1 plants. Thus, MnEIL3 may play a positive role in abiotic stress tolerances, and it indicates that the functions of EIL3/EILs in response to abiotic stresses are relatively conserved in plants.

The overexpression of kiwifruit (Actinidia deliciosa) EIN3-like transcription factors, AdEIL2 and AdEIL3, increased ethylene production by upregulating the expression of ACS and ACO genes in transgenic Arabidopsis (Yin et al., 2010). Based on the data reported by Liu et al. (2017b), several genes that were involved in ethylene biosynthesis and signal transduction showed lower expression levels in ein3-1 eill-1 plants compared with wild type Arabidopsis. Here, the transcript abundances of AtACS and AtACO genes were mainly upregulated in MnEIL3ox plants. The analysis suggested the positive feedback regulation of EIN3/EILs in ethylene production.

When plants receive the ethylene signal, EIN3/EILs are activated, and then, they regulate the transcriptional expression of downstream responsive genes, including ERF, ACO, xyloglucan endo-transglycosylase, and cell wall-modifying genes (Solano et a, 1998; Huang et al., 2010; Yin et al., 2010; Ireland et al., 2014). The PERE and ECBS motifs in promoters have been identified as EIN3-interactive motifs. MnACO1, MnACO2, MnACS1, and MnACS3 promoters contain PERE and ECBS motifs, which suggests that ethylene biosynthetic genes can be regulated by EIN3/EILs. In the present study, we found that MnEIL3 and two other MnEILs (MnEIL1 and MnEIL2) modulate the activities of MnACO1 and MnACO2 promoters as assessed by transient analysis in strawberry (Fragaria $\times$ ananassa Duch.) fruit. This result was similar to those reported in kiwifruit and melon (Cucumis melo L. cv. Andes) (Yin et al., 2010; Huang et al., 2010). MnACS1 and MnACS3 promoter' activities were regulated by MnEIL1/2/3 (Fig. 4). Thus, MnEIL proteins provide positive feedback regulation during ethylene production by directly regulating the transcription of ethylene biosynthetic genes. We also constructed MnACSIpro::GUS and MnACS3pro::GUS vectors and independently introduced them into Arabidopsis. The GUS activities in the stems and leaves of the transgenic Arabidopsis seedlings were enhanced by salt and drought stresses (Fig. 5A). Additionally, the expression levels of 
307

308

309

310

311

312

313

314

315

316

317

318

319

320

321

322

323

324

325

326

327

328

329

330

331

332

MnACS1 and MnACS3 significantly responded to salt and drought stresses (Figs. 5B-5E). The promoter' activities and gene expression levels of $\mathrm{MnACO1}$ and $\mathrm{MnACO} 2$ were also enhanced by abiotic stresses (Yu et al., 2017).

On the basis of our results, we proposed a working model for the regulatory network of mulberry MnEIL3 in response to abiotic stresses (Fig. 6). When plants are exposed to abiotic stresses, the stress signals are perceived by plant cells, leading to the enhanced accumulation of ethylene. Then, the ethylene signal transduction pathway was induced and the nucleus-localized MnEIL3 proteins' accumulation and stability were enhanced. MnEIL3's expression was also significantly induced by stresses. MnEIL3 eventually positively regulates abiotic stress tolerances by activating downstream stress-responsive genes. In addition, MnEIL3 binds to the target regions in the promoters of $A C O$ and $A C S$ genes, and activates gene expression, which contributes to the accumulation of ethylene. MnEIL1 and MnEIL2 may function in other processes, such as fruit development and maturation, by modulating ethylene responses (Liu et al., 2015). However, more work is needed to investigate the roles of the ethylene-EIN3/EILs$A C O / A C S$ regulatory loop in abiotic stress tolerances.

\section{CONCLUSIONS}

In summary, our results explored the functions of a gene encoding an EIN3-like protein from mulberry, MnEIL3. The expression level of MnEIL3 significantly increased in response to salt and drought stresses in roots and shoot. Transgenic Arabidopsis overexpressing MnEIL3 exhibited an enhanced tolerance to salt and drought stresses. The overexpression of MnEIL3 significantly upregulated the expression levels of ethylene biosynthetic genes in Arabidopsis. Moreover, MnEIL3 could enhance the activities of MnACO1 and MnACS1 promoters, which suggested an ethylene-EIN3/EILs- $A C O / A C S$ regulatory loop in abiotic stress tolerance. This research provides insights into the functions of MnEIL3 in abiotic stress tolerance and their influence on the expression levels of ethylene biosynthetic genes. 
333 We thank Dr. Hongwei Guo (Institute of Plant and Food Science, Department of Biology, 334 Southern University of Science and Technology, Shenzhen 518055, P.R. China) for providing 335 the seeds of ein3-1 eil1-1 Arabidopsis.

\section{ADDITIONAL INFORMATION AND DECLARATIONS}

\section{Funding}

338 This work was supported by the China Agriculture Research System (No. CARS-18-ZJ0201),

339 the Special Fund for Agro-scientific Research in the Public Interest of China (No. 201403064), 340 the Fundamental Research Funds for the Central Universities (No. XDJK2018C008), and the 341 National Natural Sciences Foundation of China (No. 31801126).

\section{Grant Disclosures}

343 The following grant information was disclosed by the authors:

344 The China Agriculture Research System: CARS-18-ZJ0201.

345 The Special Fund for Agro-scientific Research in the Public Interest of China: 201403064.

346 The Fundamental Research Funds for the Central Universities: XDJK2018C008.

347 The National Natural Sciences Foundation of China: 31801126.

348 Conflict of interest statement

349 The authors declare there are no competing interests.

350 Author Contributions

351 Changying Liu, Maode Yu and Aichun Zhao conceived and designed the experiments.

352 •Changying Liu, Jun Li, Panpan Zhu, Jian Yu, Jiamin Hou, and Chuanhong Wang performed 353 most of the experiments.

$354 \bullet$ Changying Liu and Dingpei Long analyzed the data.

$355 \bullet$ Changying Liu and Aichun Zhao wrote the manuscript.

356 -All the authors read and approved the final manuscript.

\section{Data Availability}

358 The following information was supplied regarding data availability:

359 The raw data has been supplied as Data S1. 
360

361

362

363

364

365

366

367

368

369

370

371

372

373

374

375

376

377

378

379

380

381

382

383

384

385

386

387

388

389

390

391

392

393

394

395

396

397

398

\section{Supplemental Information}

Supplemental information for this article can be found online at

\section{REFERENCES}

Alonso JM, Stepanova AN. 2004. The ethylene signaling pathway. Science 306:1513-1515 DOI 10.1126/science. 1104812.

Alonso JM, Stepanova AN, Solano R, Wisman E, Ferrari S, Ausubel FM, Ecker JR. 2003. Five components of the ethylene-response pathway identified in a screen for weak ethylene-insensitive mutants in Arabidopsis. Proceedings of the National Academy of Sciences of the United States of America. 100: 2992-2997 DOI 10.1073/pnas.0438070100.

An F, Zhang X, Zhu Z, Ji Y, He W, Jiang Z, Li M, Guo H. 2012. Coordinated regulation of apical hook development by gibberellins and ethylene in etiolated Arabidopsis seedlings. Cell Research 22:915-927 DOI 10.1038/cr.2012.29.

An F, Zhao Q, Ji Y, Li W, Jiang Z, Yu X, Zhang C, Han Y, He W, Liu Y, Zhang S, Ecker JR, Guo H. 2010. Ethylene-induced stabilization of ETHYLENE INSENSITIVE3 and EIN3-LIKE1 is mediated by proteasomal degradation of EIN3 binding F-box 1 and 2 that requires EIN2 in Arabidopsis. The Plant Cell 22:2384-2401 DOI 10.1105/tpc.110.076588.

An JP, Wang XF, Li YY, Song LQ, Zhao LL, You CX, Hao YJ. 2018. EIN3-LIKE1, MYB1, and ETHYLENE RESPONSE FACTOR3 act in a regulatory loop that synergistically modulates ethylene biosynthesis and anthocyanin accumulation. Plant Physiology 178:808-823 DOI 10.1104/pp.18.00068.

Chao Q, Rothenberg M, Solano R, Roman G, Terzaghi W, Ecker JR. 1997. Activation of the ethylene gas response pathway in Arabidopsis by the nuclear protein ETHYLENEINSENSITIVE3 and related proteins. Cell 89:1133-1144 DOI 10.1016/S00928674(00)80300-1.

Clark KL, Larsen PB, Wang X, Chang C. 1998. Association of the Arabidopsis CTR1 Raflike kinase with the ETR1 and ERS ethylene receptors. Proceedings of the National Academy of Sciences of the United States of America 95:5401-5406 DOI doi.org/10.1073/pnas.95.9.5401.

Clough SJ, Bent AF. 1998. Floral dip: a simplified method for Agrobacterium-mediated transformation of Arabidopsis thaliana. Plant Journal 16:735-743. DOI 10.1046/j.1365313x.1998.00343.x

Cui M, Lin Y, Zu Y, Efferth T, Li D, Tang Z. 2015. Ethylene increases accumulation of compatible solutes and decreases oxidative stress to improve plant tolerance to water stress in Arabidopsis. Journal of Plant Biology 58:193-201 DOI 10.1007/s12374-0140302-z.

He N, Zhang C, Qi X, Zhao S, Tao Y, Yang G, Lee TH, Wang X, Cai Q, Li D, Lu M, Liao S, Luo G, He R, Tan X, Xu Y, Li T, Zhao A, Jia L, Fu Q, Zeng Q, Gao C, Ma B, Liang J, Wang X, Shang J, Song P, Wu H, Fan L, Wang Q, Shuai Q, Zhu J, Wei C, ZhuSalzman K, Jin D, Wang J, Liu T, Yu M, Tang C, Wang Z, Dai F, Chen J, Liu Y, 
399

400

401

402

403

404

405

406

407

408

409

410

411

412

413

414

415

416

417

418

419

420

421

422

423

424

425

426

427

428

429

430

431

432

433

434

435

436

437

438

439

Zhao S, Lin T, Zhang S, Wang J, Wang J, Yang H, Yang G, Wang J, Paterson AH, Xia Q, Ji D, Xiang Z. 2013. Draft genome sequence of the mulberry tree Morus notabilis. Nature communications 4:2445 DOI 10.1038/Ncomms3445.

He W, Brumos J, Li H, Ji Y, Ke M, Gong X, Zeng Q, Li W, Zhang X, An F, Wen X, Li P, Chu J, Sun X, Yan C, Yan N, Xie DY, Raikhel N, Yang Z, Stepanova AN, Alonso JM, Guo H. 2011. A small-molecule screen identifies L-kynurenine as a competitive inhibitor of TAA1/TAR activity in ethylene-directed auxin biosynthesis and root growth in Arabidopsis. The Plant Cell 23:3944-3960 DOI 10.1105/tpc.111.089029.

Hua J, Meyerowitz EM. 1998. thylene responses are negatively regulated by a receptor gene family in Arabidopsis thaliana. Cell 94:261-271 DOI 10.1016/S0092-8674(00)81425-7.

Huang S, Sawaki T, Takahashi A, Mizuno S, Takezawa K, Matsumura A, Yokotsuka M, Hirasawa Y, Sonoda M, Nakagawa H, Sato T. 2010. Melon EIN3-like transcription factors (CmEIL1 and CmEIL2) are positive regulators of an ethylene- and ripeninginduced 1-aminocyclopropane-1-carboxylic acid oxidase gene (CM-ACO1). Plant Science 178:251-257 DOI 10.1016/j.plantsci.2010.01.005.

Ireland HS, Gunaseelan K, Muddumage R, Tacken EJ, Putterill J, Johnston JW, Schaffer RJ. 2014. Ethylene regulates apple (Malus $x$ domestica) fruit softening through a dose $\mathrm{x}$ time-dependent mechanism and through differential sensitivities and dependencies of cell wall-modifying genes. Plant and Cell Physiology 55:1005-1016 DOI 10.1093/pcp/pcu034.

Jefferson RA. 1987. Assaying chimeric genes in plants: The GUS gene fusion system. Plant Molecular Biology Reporter 5:387-405

Kende H. 1993. Ethylene biosynthesis. Annual Review of Plant Physiology and Plant Molecular Biology 44:283-307 DOI 10.1146/annurev.pp.44.060193.001435.

Kim GD, Cho YH, Yoo SD. 2017. Regulatory functions of cellular energy sensor SNF1-related kinase1 for leaf senescence delay through ETHYLENE-INSENSITIVE3 repression. Scientific Reports 7:3193 DOI 10.1038/S41598-017-03506-1.

Kong X, Li C, Zhang F, Yu Q, Gao S, Zhang M, Tian H, Zhang J, Yuan X, Ding Z. 2018. Ethylene promotes cadmium-induced root growth inhibition through EIN3 controlled XTH33 and LSU1 expression in Arabidopsis. Plant Cell and Environment 41:2449-2462 DOI 10.1111/pce.13361.

Liu CY, Lu RH, Li J, Zhao AC, Wang XL, Diane U, Wang XH, Wang CH, Yu YS, Han SM, Lu C, Yu MD. 2014. Characterization and expression profiles of MaACS and MaACO genes from mulberry (Morus alba L.). Journal of Zhejiang University-SCIENCE B 15:611-623 DOI 10.1631/jzus.B1300320.

Liu C, Wei C, Zhang M, Xu Y, Xiang Z, Zhao A. 2017a. Mulberry MnMAPKl, a group C mitogen-activated protein kinase gene, endowed transgenic Arabidopsis with novel responses to various abiotic stresses. Plant Cell, Tissue and Organ Culture 131:151-162 DOI 10.1007/s11240-017-1272-x.

Liu C, Zhao A, Zhu P, Li J, Han L, Wang X, Fan W, Lu R, Wang C, Li Z, Lu C, Yu M. 2015. Characterization and expression of genes involved in the ethylene biosynthesis and 
440

441

442

443

444

445

446

447

448

449

450

451

452

453

454

455

456

457

458

459

460

461

462

463

464

465

466

467

468

469

470

471

472

473

474

475

476

477

478

479

480

signal transduction during ripening of mulberry fruit. Plos One 10:e0122081 DOI 10.1371/journal.pone.0122081.

Liu X, Liu R, Li Y, Shen X, Zhong S, Shi H. 2017b. EIN3 and PIF3 form an interdependent module that represses chloroplast development in buried seedlings. The Plant Cell 29:3051-3067 DOI 10.1105/tpc.17.00508.

Peng JY, Li ZH, Wen X, Li WY, Shi H, Yang LS, Zhu HQ, Guo HW. 2014. Salt-induced stabilization of EIN3/EIL1 confers salinity tolerance by deterring ROS accumulation in Arabidopsis. Plos Genetics 10:e1004664 DOI 10.1371/journal.pgen.1004664.

Qiao H, Shen Z, Huang SS, Schmitz RJ, Urich MA, Briggs SP, Ecker JR. 2012. Processing and subcellular trafficking of ER-tethered EIN2 control response to ethylene gas. Science 338:390-393. DOI 10.1126/science.1225974.

Potuschak T, Lechner E, Parmentier Y, Yanagisawa S, Grava S, Koncz C, Genschik P. 2003. EIN3-dependent regulation of plant ethylene hormone signaling by two Arabidopsis F box proteins: EBF1 and EBF2. Cell 115:679-689 DOI 10.1016/S00928674(03)00968-1.

Rodríguez FI, Esch JJ, Hall AE, Binder BM, Schaller GE, Bleecker AB. 1999. A copper cofactor for the ethylene receptor ETR1 from Arabidopsis. Science 283:996-998 DOI 10.1126/science.283.5404.996.

Shan W, Kuang JF, Chen L, Xie H, Peng HH, Xiao YY, Li XP, Chen WX, He QG, Chen JY, Lu WJ. 2012. Molecular characterization of banana NAC transcription factors and their interactions with ethylene signalling component EIL during fruit ripening. Journal of Experimental Botany 63:5171-5187 DOI 10.1093/jxb/ers178.

Shi H, Lyu M, Luo YW, Liu SC, Li Y, He H, Wei N, Deng XW, Zhong S. 2018. Genomewide regulation of light-controlled seedling morphogenesis by three families of transcription factors. Proceedings of the National Academy of Sciences of the United States of America 115:6482-6487 DOI 10.1073/pnas.1803861115.

Shi Y, Tian S, Hou L, Huang X, Zhang X, Guo H, Yang S. 2012. Ethylene signaling negatively regulates freezing tolerance by repressing expression of CBF and type-A ARR genes in Arabidopsis. The Plant Cell 24:2578-2595. DOI 10.1105/tpc.112.098640.

Solano R, Stepanova A, Chao QM, Ecker JR. 1998. Nuclear events in ethylene signaling: a transcriptional cascade mediated by ETHYLENE-INSENSITIVE3 and ETHYLENERESPONSE-FACTOR1. Gene and Development 12:3703-3714 DOI 10.1101/gad.12.23.3703.

Spolaore S, Trainotti L, Casadoro G. 2001. A simple protocol for transient gene expression in ripe fleshy fruit mediated by Agrobacterium. Journal of Experimental Botany 52:845-850 DOI 10.1093/jexbot/52.357.845.

Wang KLC, Li H, Ecker JR. 2002. Ethylene biosynthesis and signaling networks. The Plant Cell 14:S131-S151 DOI 10.1105/tpc.001768.

Wei C, Liu X, Long D, Guo Q, Fang Y, Bian C, Zhang D, Zeng Q, Xiang Z, Zhao A. 2014. Molecular cloning and expression analysis of mulberry $M A P K$ gene family. Plant Physiology and Biochemistry 77:108-116 DOI 10.1016/j.plaphy.2014.02.002. 
481

482

483

484

485

486

487

488

489

490

491

492

493

494

495

496

497

498

499

500

501

502

503

504

505

506

507
Yang SF, Hoffman NE. 1984. Ethylene biosynthesis and its regulation in higher-plants. Annual Review of Plant Physiology 35:155-189 DOI 10.1146/annurev.pp.35.060184.001103.

Yin XR, Allan AC, Chen KS, Ferguson IB. 2010. Kiwifruit $E I L$ and $E R F$ genes involved in regulating fruit ripening. Plant Physiology 153:1280-1292 DOI 10.1104/pp.110.157081.

Yoo SD, Cho YH, Tena G, Xiong Y, Sheen J. 2008. Dual control of nuclear EIN3 by bifurcate MAPK cascades in $\mathrm{C}_{2} \mathrm{H}_{4}$ signalling. Nature 451:789-795 DOI 10.1038/nature06543.

Yu J, Liu CY, Zhao AC, Wang CH, Cai YX, Yu MD. 2017. Functional analysis of 1aminocyclopropane-1-carboxylate oxidase gene's promoter in mulberry. Acta Agronomica Sinica 43:839-848 DOI 10.3724/SP.J.1006.2017.00839.

Yu Y, Wang J, Shi H, Gu J, Dong J, Deng XW, Huang R. 2016. Salt stress and ethylene antagonistically regulate nucleocytoplasmic partitioning of COP1 to control seed germination. Plant Physiology 170:2340-2350 DOI 10.1104/pp.15.01724.

Yu Y, Wang J, Zhang Z, Quan R, Zhang H, Deng XW, Ma L, Huang R. 2013. Ethylene promotes hypocotyl growth and HY5 degradation by enhancing the movement of COP1 to the nucleus in the light. Plos Genetics 9:e1004025 DOI 10.1371/journal.pgen.1004025.

Zhang L, Li Z, Quan R, Li G, Wang R, Huang R. 2011. An AP2 domain-containing gene, ESE1, targeted by the ethylene signaling component EIN3 is important for the salt response in Arabidopsis. Plant Physiology 157:854-865 DOI 10.1104/pp.111.179028.

Zhong S, Shi H, Xue C, Wei N, Guo H, Deng XW. 2014. Ethylene-orchestrated circuitry coordinates a seedling's response to soil cover and etiolated growth. Proceedings of the National Academy of Sciences of the United States of America 111:3913-3920 DOI 10.1073/pnas.1402491111.

Zhu Z, An F, Feng Y, Li P, Xue L, A M, Jiang Z, Kim JM, To TK, Li W, Zhang X, Yu Q, Dong Z, Chen WQ, Seki M, Zhou JM, Guo H. 2011. Derepression of ethylenestabilized transcription factors (EIN3/EIL1) mediates jasmonate and ethylene signaling synergy in Arabidopsis. Proceedings of the National Academy of Sciences of the United States of America 108:12539-12544 DOI 10.1073/pnas.1103959108. 
510 Figure 1 Expression profiles of MnEIL genes in response to salt and drought stresses. Mulberry 511 seedlings were subjected to salt $[0.6 \%(\mathrm{~m} / \mathrm{v}) \mathrm{NaCl}]$ and drought $[20 \%(\mathrm{~m} / \mathrm{v})$ PEG6000]. (A) 512 MnEIL gene expression levels in roots under salt stress. (B) MnEIL gene expression levels in 513 shoots under salt stress. (C) MnEIL gene expression levels in roots under drought stress. (D) 514 MnEIL gene expression levels in shoots under drought stress. Data are means \pm SEs $(\mathrm{n}=3)$. 515 Means within a column with different letters are significantly different $(P<0.05)$. Means within a 516 column with the same letters are no significant different $(P>0.05)$.

517 Figure 2 Stress tolerance analyses of wild type, MnEIL3ox, and ein3-1 eill-1 Arabidopsis. (A) 518 The growth of wild type, MnEIL3ox, and ein3-1 eil1-1 Arabidopsis plants under normal, salt and 519 drought conditions. (B-E) The survival rates (B), MDA content (C), $\mathrm{H}_{2} \mathrm{O}_{2}$ content (D), and 520 proline content (E) of wild type, MnEIL3ox, and ein3-1 eil1-1 Arabidopsi plants under normal 521 and stress conditions. Data are means \pm SEs $(n=3)$. Means within a column with different letters 522 are significantly different $(P<0.05)$. Means within a column with the same letters are no 523 significant different $(P>0.05)$.

524 Figure 3 qRT-PCR analysis of changes in AtACS and AtACO genes in MnEIL3ox plants. Data 525 are means \pm SEs $(n=3)$. Significant differences $(P<0.05)$ are marked with asterisks. The gene 526 expression in wild type was set as 1.

527 Figure 4 In vivo interactions of MnEIL3 with ethylene biosythetic genes promoters. (A) GUS staining of the injected strawberry fruit. (B) The detection of GUS activities. Data are means \pm SEs $(\mathrm{n}=3)$. Significant differences $(P<0.05)$ are marked with asterisks. Figure 5 Analyses of promoter activities and expression levels of MnACS1 and MaACS3 genes 531 in response to salt and drought treatments. (A) Histochemical GUS staining of the 532 MnACS1pro::GUS and MnACS3pro::GUS transgenic Arabidopsis under salt [200 mM NaCl] 533 and drought (200 mM mannitol) treatments. Scale bar, $2 \mathrm{~mm}$. (B) The expression level of 534 MnACS1 in response to salt treatment. (C) The expression level of MnACS1 in response to 535 drought treatment. (D) The expression level of MnACS3 in response to salt treatment. (E) The 
536 expression level of MnACS3 in response to drought treatment. Data are means \pm SEs $(n=3)$. 537 Means within a column with different letters are significantly different $(P<0.05)$. Means within a 538 column with the same letters are no significant different $(P>0.05)$.

539 Figure 6 A possible model of MnEIL3 in responses to abiotic stresses. 


\section{Figure 1}

Figure 1

Expression profiles of MnEIL genes in response to salt and drought stresses. Mulberry seedlings were subjected to salt [0.6\% (m/v) NaCl] and drought [20\% (m/v) PEG6000]. (A) MnEIL gene expression levels in roots under salt stress. (B) MnEIL gene expression levels in shoots under salt stress. (C) MnEIL gene expression levels in roots under drought stress. (D) MnEIL gene expression levels in shoots under drought stress. Data are means \pm SEs $(n=3)$. Means within a column with different letters are significantly different $(P<0.05)$. Means within a column with the same letters are no significant different $(P>0.05)$. 


$$
\text { A }
$$

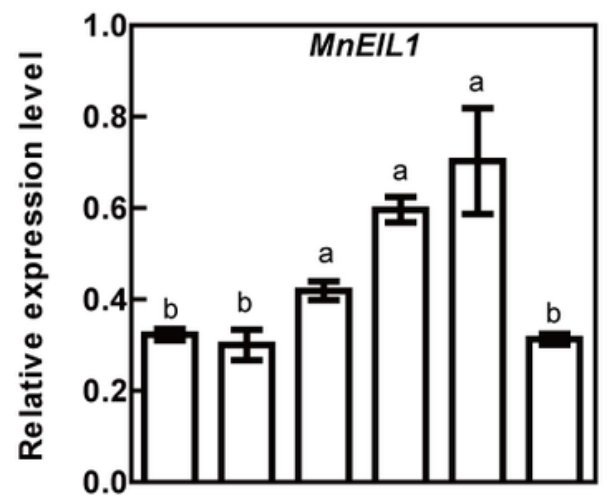

B

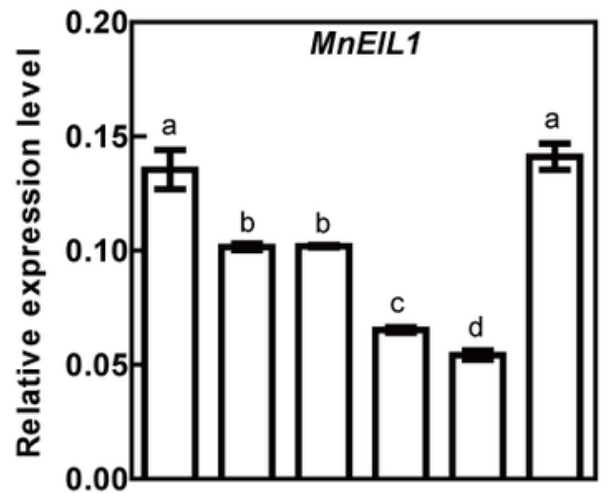

C

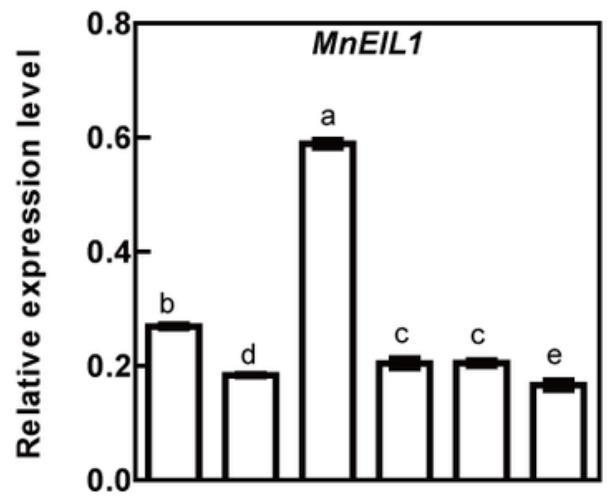

D

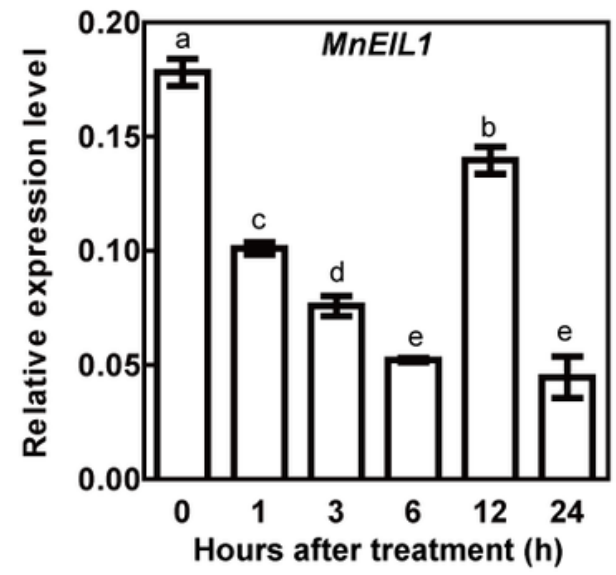

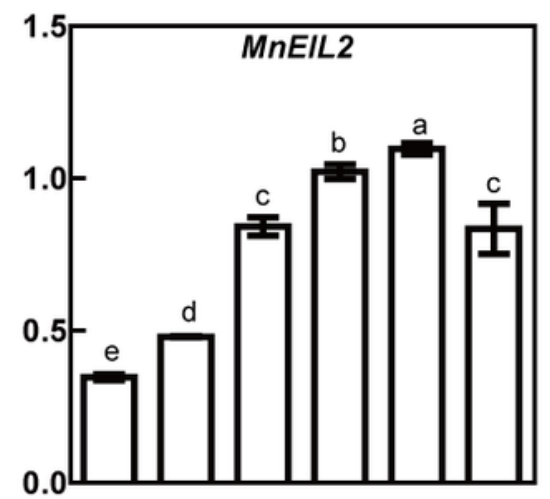
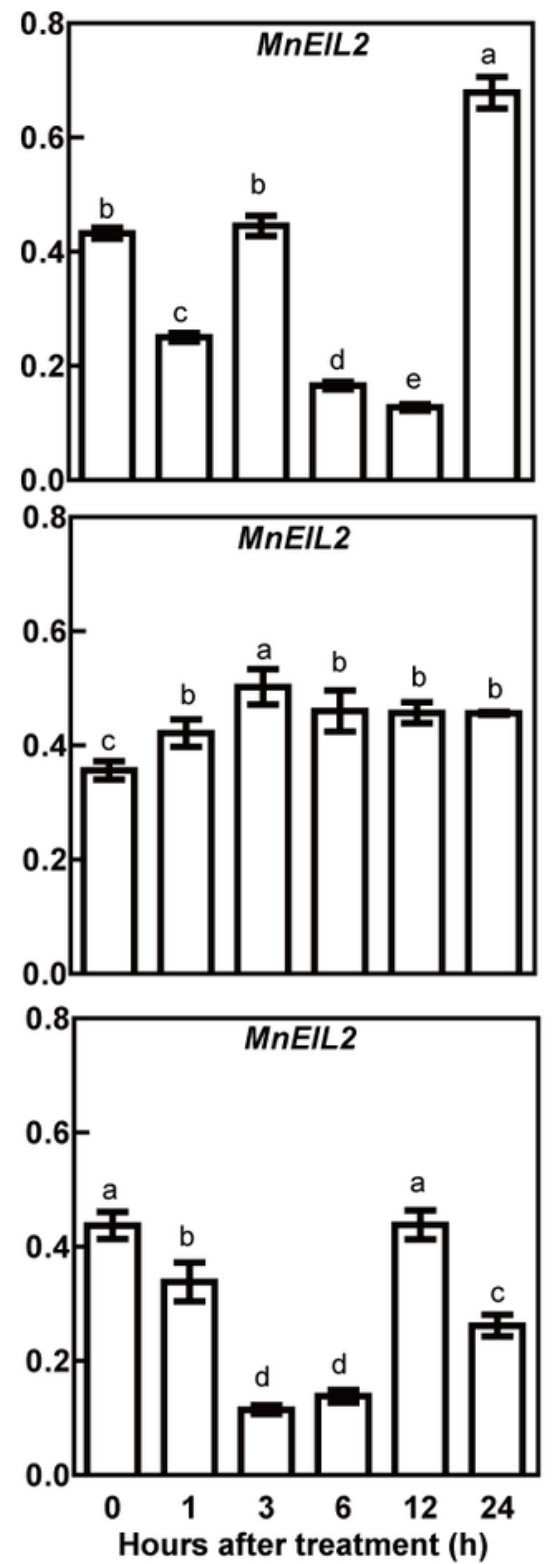
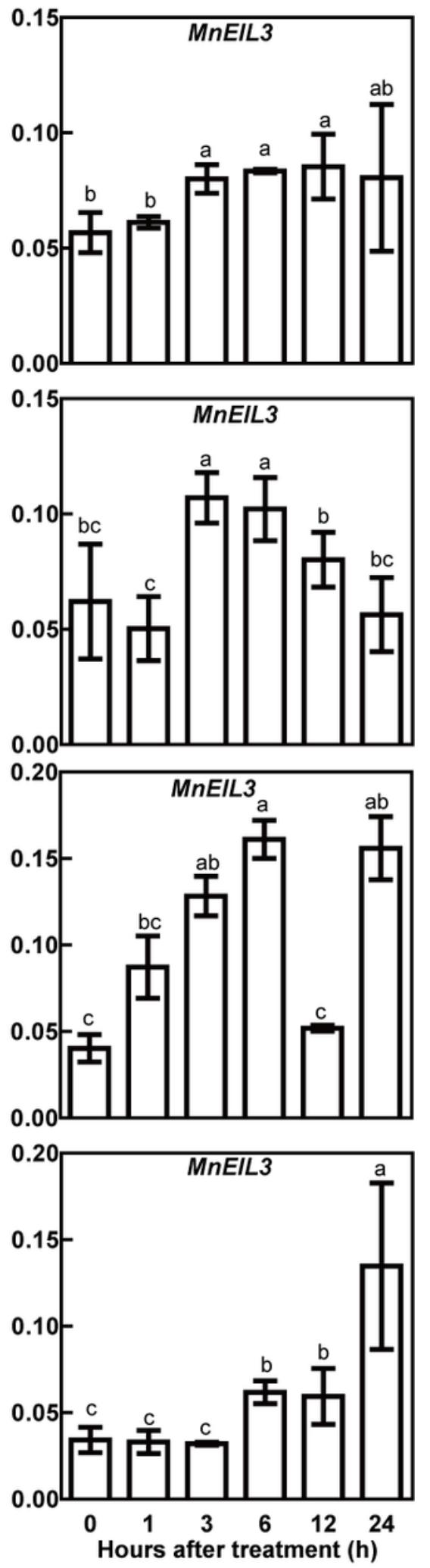


\section{Figure 2}

Figure 2

Stress tolerance analyses of wild type, MnEIL3ox, and ein3-1 eil1-1 Arabidopsis. (A) The growth of wild type, MnEIL3ox, and ein3-1 eil1-1 Arabidopsis plants under normal, salt and drought conditions. (B-E) The survival rates (B), MDA content (C), $\mathrm{H}_{2} \mathrm{O}_{2}$ content (D), and proline content (E) of wild type, MnEIL3ox, and ein3-1 eil1-1 Arabidopsi plants under normal and stress conditions. Data are means \pm SEs $(n=3)$. Means within a column with different letters are significantly different $(P<0.05)$. Means within a column with the same letters are no significant different $(P>0.05)$.

A

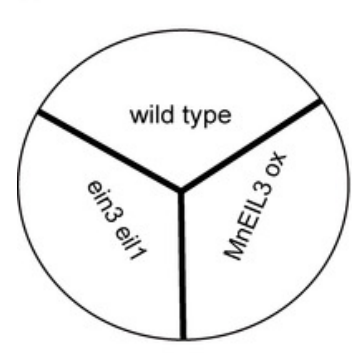

B

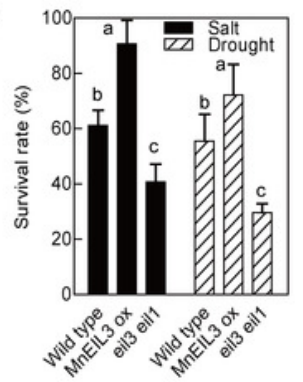

without stress

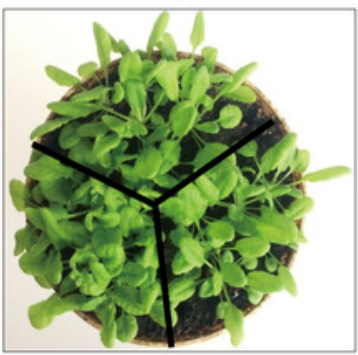

C

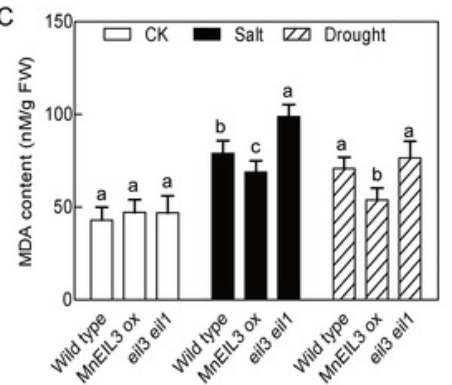

$1.2 \% \mathrm{NaCl}$

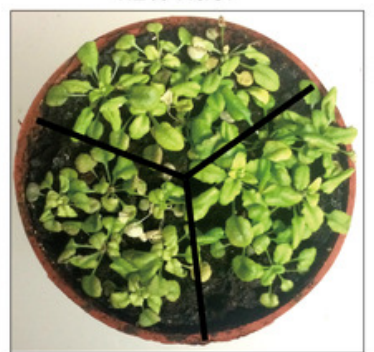

Drought stress

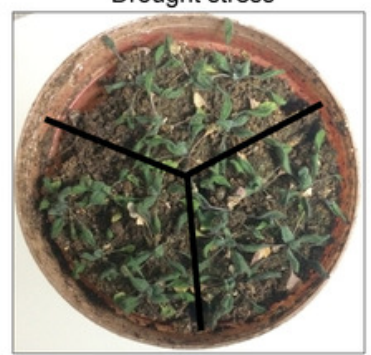

$3 \mathrm{~d}$ after rewatering

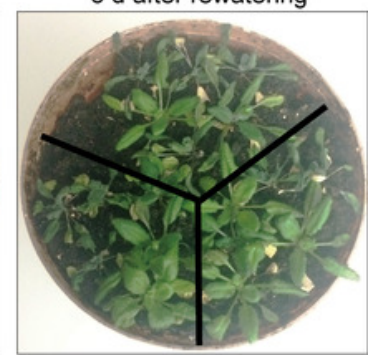

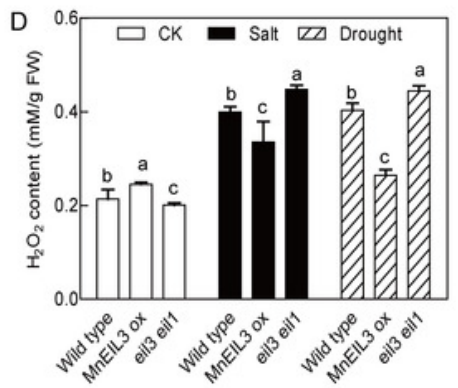

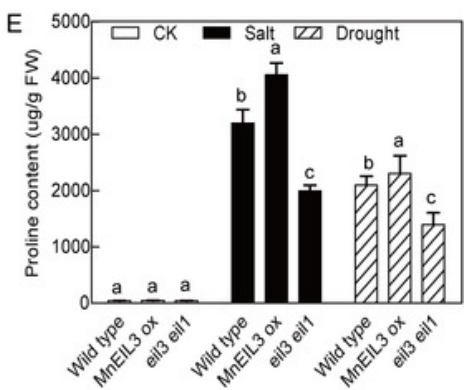


Figure 3

Figure 3

qRT-PCR analysis of changes in AtACS and AtACO genes in MnEIL3ox plants. Data are means

\pm SEs $(n=3)$. Significant differences $(P<0.05)$ are marked with asterisks. The gene expression in wild type was set as 1 .

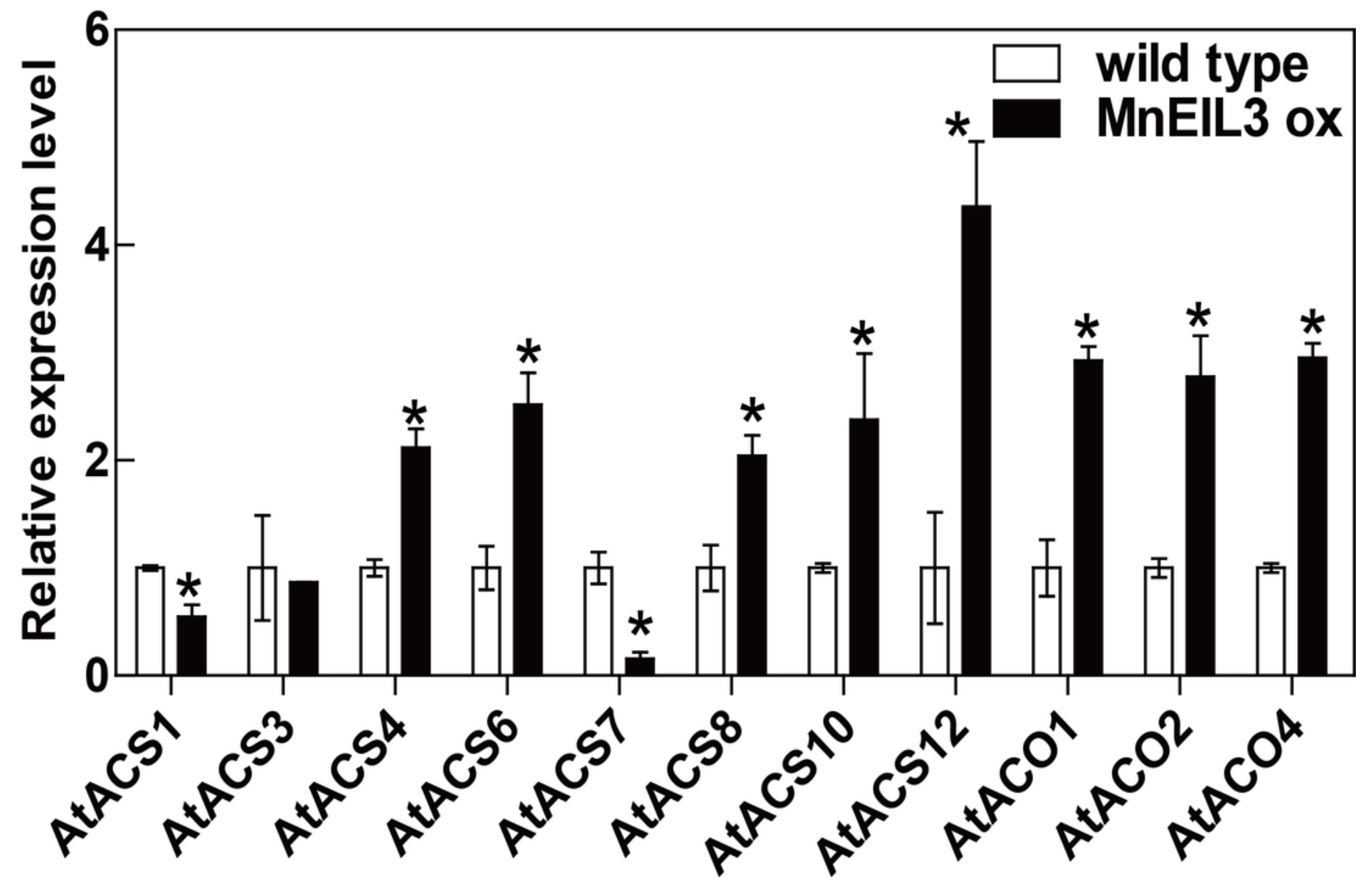




\section{Figure 4}

Figure 4

In vivo interactions of MnEIL3 with ethylene biosythetic genes promoters. (A) GUS staining of the injected strawberry fruit. (B) The detection of GUS activities. Data are means \pm SEs $(n=3)$. Significant differences $(P<0.05)$ are marked with asterisks. 


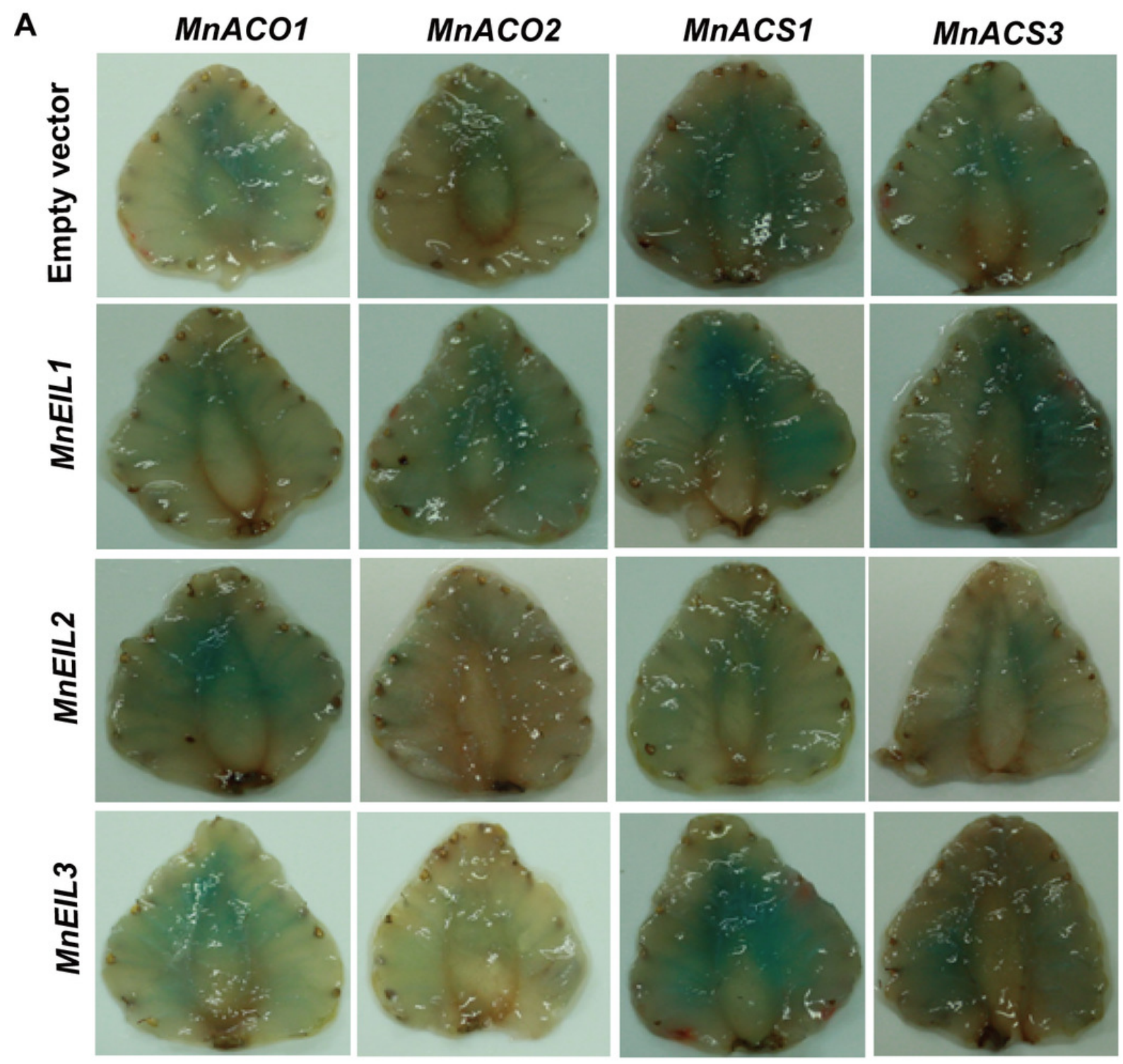

B

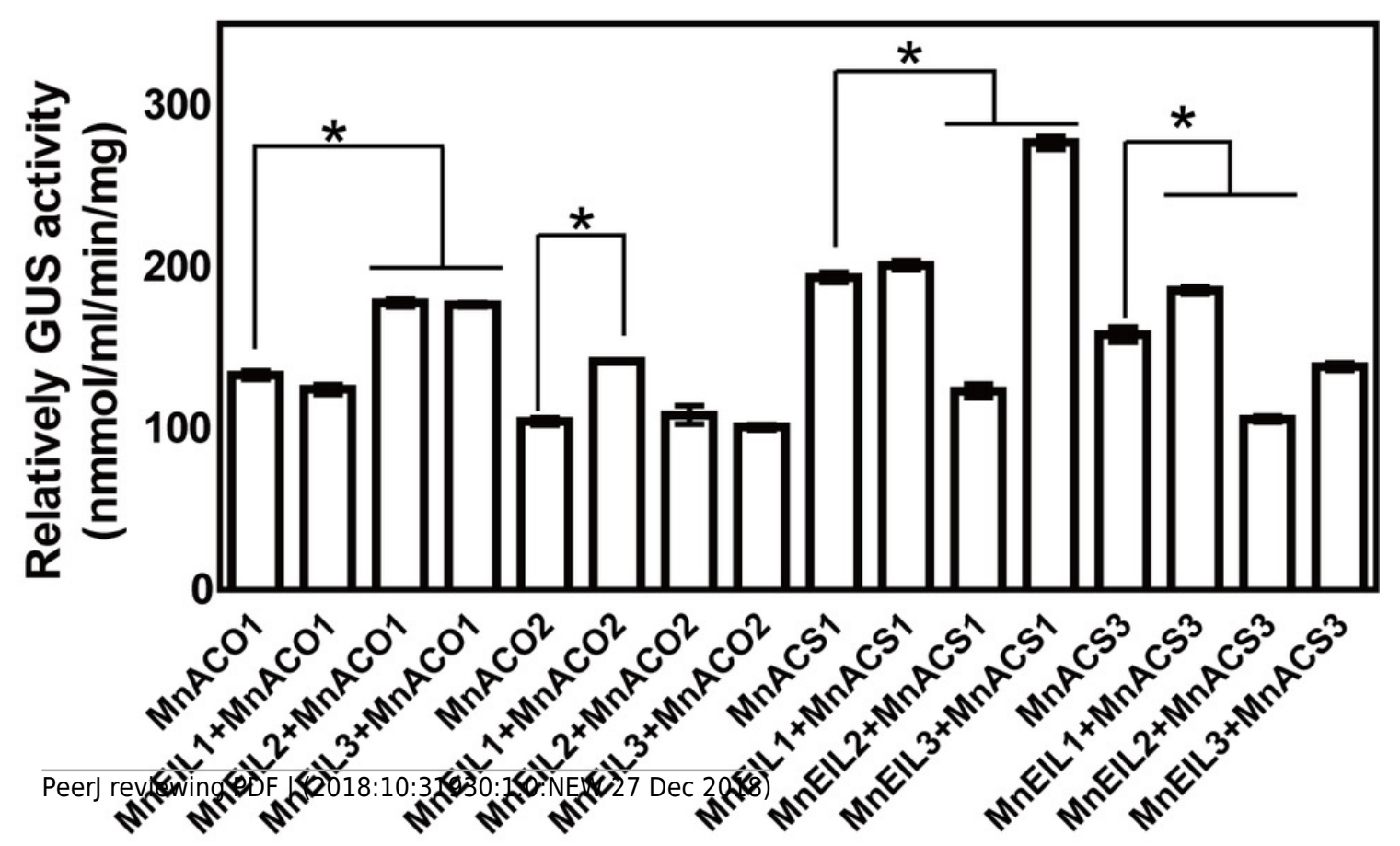




\section{Figure 5}

Figure 5

Analyses of promoter activities and expression levels of MnACS1 and MaACS3 genes in response to salt and drought treatments. (A) Histochemical GUS staining of the MnACS1pro::GUS and MnACS3pro::GUS transgenic Arabidopsis under salt [200 mM NaCl] and drought (200 mM mannitol) treatments. Scale bar, $2 \mathrm{~mm}$. (B) The expression level of MnACS1 in response to salt treatment. (C) The expression level of MnACS1 in response to drought treatment. (D) The expression level of MnACS3 in response to salt treatment. (E) The expression level of MnACS3 in response to drought treatment. Data are means \pm SEs $(n=3)$. Means within a column with different letters are significantly different $(P<0.05)$. Means within a column with the same letters are no significant different $(P>0.05)$.
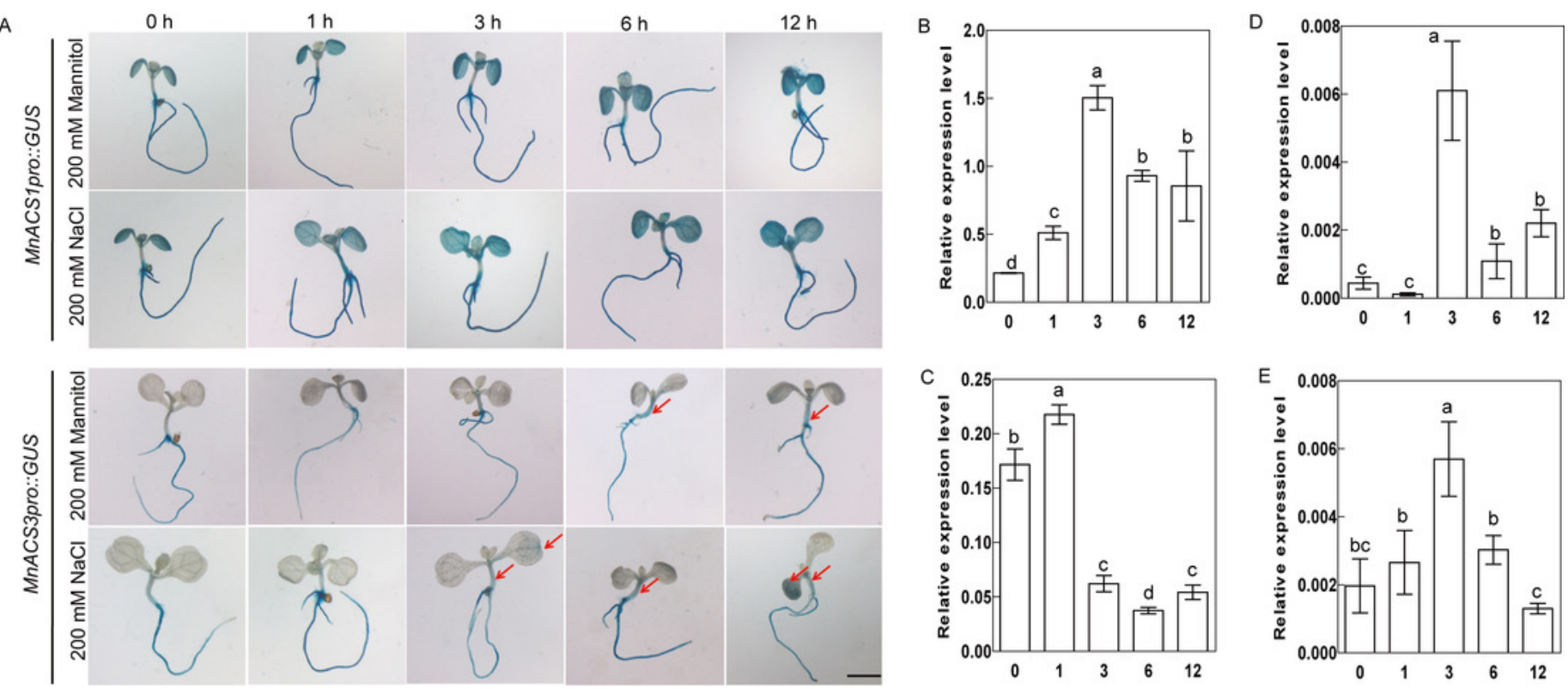
Figure 6

Figure 6

A possible model of MnEIL3 in responses to abiotic stresses.

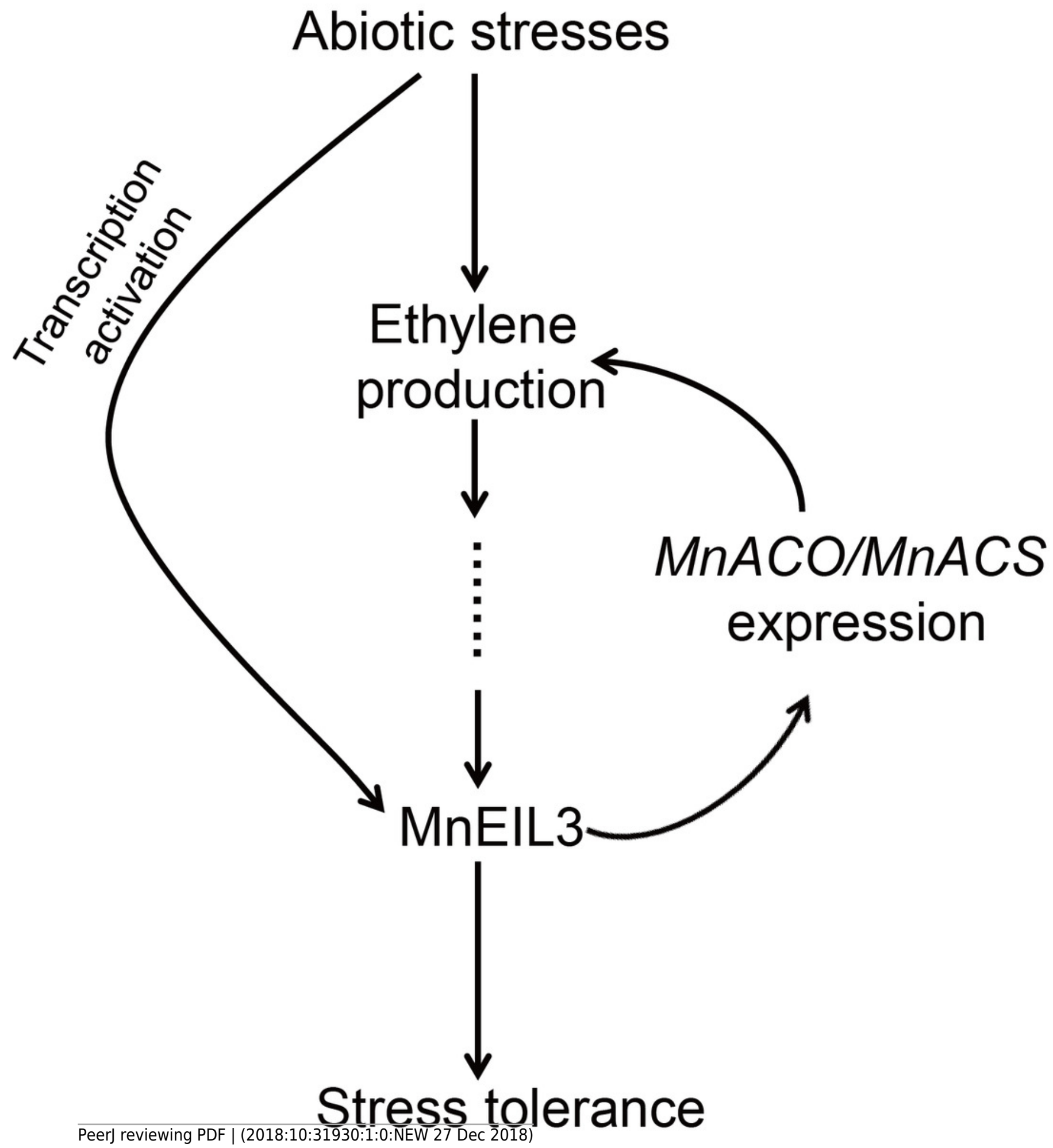

